\title{
Adequação da formação em saúde do adolescente: opiniões dos médicos e enfermeiros de família que frequentaram uma ação formativa
}

Maria Inês Santos, ${ }^{1}$ Frederico Rosário, ${ }^{2}$ Alzira Ferrão, ${ }^{3}$ Elisabete Santos ${ }^{4}$

\begin{abstract}
RESUMO
Objetivos: Avaliar a perceção que os profissionais de saúde dos cuidados de saúde primários têm sobre a formação recebida em saúde do adolescente e identificar as limitações sentidas e as necessidades de formação adicional.

Tipo de estudo: Estudo observacional, transversal e descritivo.

Local: Região Centro.

População: Médicos e enfermeiros de família da região Centro.

Métodos: Os médicos e enfermeiros de família inscritos numa formação de saúde do adolescente foram convidados a responder a um inquérito em que se solicitava a avaliação da sua formação pré e pós-graduada em saúde do adolescente, a necessidade de receber formação adicional e as limitações.

Resultados: A maioria dos participantes considerou que a sua formação pré-graduada era insuficiente (médicos: $97 \%$; enfermeiros: $77,2 \%$ ) e que não se sentia apto para lidar com adolescentes após a licenciatura (médicos: 93,9\%; enfermeiros: 87,7\%). A maioria dos médicos de família também considerou insuficiente a formação recebida durante o internato $(77,5 \%)$, não se sentindo aptos a lidar com adolescentes no final do mesmo $(68,4 \%)$, tendo ainda considerado insuficiente a formação frequentada após o internato $(73,0 \%)$. Os participantes consideraram ter necessidade de receber formação adicional em saúde do adolescente, apontando áreas com maiores necessidades formativas: as queixas psicossomáticas $(82,2 \%)$, os problemas familiares $(72,9 \%)$, a entrevista ao adolescente (69,5\%), o consumo de substâncias $(62,6 \%)$ e os problemas escolares e de comportamento $(62,3 \%)$. Conclusões: Os médicos e enfermeiros de família inquiridos referiram sentir importantes lacunas formativas em saúde do adolescente na sua formação pré e pós-graduada, bem como a necessidade de obter mais formação nesta área.
\end{abstract}

Palavras-chave: Educação; Saúde do adolescente; Cuidados de saúde primários

\section{INTRODUÇÃO}

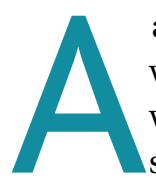
adolescência é um período fulcral no desenvolvimento do ser humano. É nesta fase da vida que se observam as maiores alterações fisiológicas, biológicas e sociais ${ }^{1-2}$ que permitem a transformação da criança num adulto autónomo, capaz de se integrar e interagir com a sociedade. ${ }^{3-4} \mathrm{E}$ também a fase com maior potencial para a adoção de estilos de vida saudáveis, uma vez que a sua assimilação nesta idade condiciona a sua manutenção na vida adulta na maioria dos casos. ${ }^{3-4}$
A promoção de estilos de vida saudáveis no adolescente assume-se como uma das atividades clínicas com melhor relação benefício-custo. ${ }^{5}$ Está provado que todo o investimento realizado neste grupo etário, em termos

\footnotetext{
1. Médica assistente de Pediatria. Unidade de Medicina do Adolescente, Centro Hospitalar Tondela-Viseu, E.P.E., Viseu, Portugal.

2. Médico assistente de Medicina Geral e Familiar. Unidade de Cuidados de Saúde Personalizados Tomaz Ribeiro - Extensão de Molelos, Tondela, Portugal.

3. Médica assistente graduada sénior. Diretora do Serviço de Pediatria, Centro Hospitalar Tondela-Viseu, E.P.E., Viseu, Portugal.

4. Médica assistente graduada de Pediatria. Coordenadora da Unidade de Medicina do Adolescente, Centro Hospitalar Tondela-Viseu, E.P.E., Viseu, Portugal.
} 
de redução de fatores de risco e de promoção de fatores protetores, tem como retorno ganhos efetivos em saúde. Esta relação benefício-custo é francamente superior às mesmas atividades realizadas nos grupos etários seguintes, uma vez que a maior parte delas apenas permite diminuir as consequências da doença. ${ }^{6-8} \mathrm{Em}$ Portugal, estas atividades preventivas assumem o seu expoente máximo ao nível dos cuidados de saúde primários, uma vez que é neste contexto que a maioria dos adolescentes é acompanhada. ${ }^{9}$

Os profissionais de saúde dos cuidados de saúde primários encontram-se, portanto, em posição privilegiada para desenvolver atividades preventivas com os adolescentes. ${ }^{10-11} \mathrm{~A}$ evidência mostra que os adolescentes aconselhados por estes profissionais apresentam uma menor incidência de comportamentos de risco quando comparados com adolescentes não aconselhados. ${ }^{12}$ O efeito favorável do aconselhamento ao nível dos cuidados de saúde primários em conjunto com o facto dos adolescentes constituírem cerca de $10 \%$ da população residente em Portugal ${ }^{13}$ torna crucial que os profissionais de saúde estejam preparados para atender este grupo etário. ${ }^{1}$

A evidência mostra uma relação positiva entre o treino dos médicos em saúde do adolescente e a melhoria dos cuidados de saúde ${ }^{14-16}$ pelo que várias sociedades internacionais têm recomendado um acréscimo de formação nesta área. ${ }^{16-21}$ Apesar de haver uma preocupação crescente com a saúde do adolescente, a formação nesta área é deficiente. ${ }^{8,16,22-26}$

Em Portugal, a formação pré-graduada em saúde do adolescente é ministrada no contexto das cadeiras de pediatria e, como tal, tem habitualmente uma duração relativamente limitada. ${ }^{27}$ Para além da formação prégraduada, os médicos de família contactam com a saúde do adolescente durante o estágio de Saúde Infantil e Juvenil, no Internato do Ano Comum e no Internato de Formação Específica e, ainda, nos seus estágios de medicina geral e familiar. Estes estágios, que têm durações curtas, incluem a frequência de várias outras valências, pelo que o contato com o adolescente é, na maioria dos casos, esporádico e não sistematizado ${ }^{28}$. Também não existem estudos no nosso país que documentem o nível de preparação dos profissionais de saúde dos cuidados de saúde primários nesta área específica ou as dificuldades por eles sentidas quando tentam abordar os problemas do adolescente.

Assim, este estudo tem como objetivos avaliar a perceção que os profissionais de saúde dos cuidados de saúde primários têm sobre a formação recebida em saúde do adolescente e identificar as limitações sentidas e as necessidades de formação adicional nesta área.

\section{MÉTODO}

\section{Contexto}

Entre setembro e outubro de 2014, a Administração Regional de Saúde da Região Centro promoveu seis ações de formação em saúde do adolescente dirigidas a médicos de família, médicos internos de medicina geral e familiar e enfermeiros de família dos seis ACeS da Região Centro (ACeS Pinhal Litoral, Dão Lafões, Baixo Vouga, Cova da Beira, Pinhal Interior Norte e Baixo Mondego). Foi enviada ao coordenador de cada Unidade de Saúde dos respetivos ACeS uma carta a explicar, de forma resumida, o objetivo da formação e a convidá-lo a selecionar da respetiva Unidade de Saúde, preferencialmente, um médico e um enfermeiro para participar na formação.

\section{Tipo de estudo e amostra}

Estudo observacional, transversal, descritivo.

Amostra de conveniência, constituída pelos médicos e enfermeiros de família que foram convidados para participar numa ação de formação em saúde do adolescente. Não foi aplicado qualquer critério de exclusão.

\section{Instrumento de medida}

Na fase pré-formação, os participantes foram convidados a preencher um questionário (Anexo), elaborado com base na revisão da literatura ${ }^{2-3,9-10,16,23-24,29} \mathrm{e}$ especificamente desenhado para o efeito dada a inexistência de questionários validados nesta área. O questionário pedia aos participantes informação sobre dados demográficos, sobre formação específica em saúde do adolescente, a sua perceção da atual adequação do treino recebido, qual o grupo etário em idade pediátrica com que preferiam trabalhar (recém-nascido/lactente, criança ou adolescente), sobre limitações que sentiam na sua formação em saúde do adolescente e em que áreas gostariam de ter formação adicional.

A adequação da formação pré-graduada e a necessidade de formação adicional foi avaliada com base no 
grau de concordância relativamente a três afirmações, medida numa escala de Likert de sete pontos, em que «1» representava total discordância e «7» total concordância. A formação pós-graduada também foi avaliada com base no grau de concordância a três afirmações, medida numa escala de Likert de sete pontos. Estes três últimos itens solicitavam informação sobre a formação recebida durante o internato médico, pelo que apenas se consideraram as respostas dos médicos especialistas em medicina geral e familiar.

Para a identificação das limitações e necessidades adicionais de formação foi solicitado aos participantes que respondessem «Sim» ou «Não» em relação às suas limitações e/ou necessidade de treino adicional em cada uma de nove grandes áreas da saúde do adolescente: entrevista ao adolescente; problemas do desenvolvimento e crescimento (físico, social e psicológico); alimentação, obesidade e perturbações do comportamento alimentar; sexualidade, gravidez e comportamentos sexuais de risco; consumo de substância psicoativas (tabaco, álcool e outras substâncias); família: dinâmicas, adulto de referência e problemas familiares; problemas escolares e de comportamento; queixas psicossomáticas, depressão e risco de suicídio; comportamentos de risco e acidentes.

\section{Considerações éticas}

A primeira página do questionário continha uma explicação breve do estudo e dos seus objetivos. Ao participante foi solicitado o seu consentimento informado e garantida a confidencialidade dos dados. O protocolo do estudo foi aprovado pela Comissão de Ética da Administração Regional de Saúde do Centro.

\section{Análise dos dados}

Os dados recolhidos foram introduzidos pelo investigador principal (MIS), tendo a cada participante sido atribuído um código apenas do conhecimento deste investigador. Os dados foram depois analisados por um segundo elemento da equipa de investigação (FR) que não teve acesso à chave que permitia a sua identificação. Para efeitos de análise estatística procedeu-se à transformação das seguintes variáveis: os grupos profissionais foram dicotomizados em médicos e enfermeiros; a preferência por grupo etário foi dicotomizada em adolescentes e outros; os itens relativos à for- mação pré e pós-graduada foram dicotomizados em «Concordo» para pontuações superiores a quatro e em «Discordo» para pontuações menores ou iguais a quatro.

Os resultados são apresentados como média \pm desvio-padrão para variáveis contínuas e distribuição de frequências para as variáveis categóricas. Os dados foram analisados com o programa $\mathrm{IBM}^{\odot}$ SPSS $^{\odot}$ Statistics v. 22 .

\section{RESULTADOS}

\section{Características dos participantes}

Dos 90 profissionais de saúde que participaram neste estudo, 57 (63,3\%) eram enfermeiros (Quadro I). Os participantes tinham em média $42,3 \pm 9,8$ anos, sendo 83 $(92,2 \%)$ do sexo feminino.

\section{Formação e grupo etário preferido}

A maioria dos participantes considerou que a formação recebida durante a faculdade foi insuficiente (médicos: 97,0\%; enfermeiros: $77,2 \%$ ) e que não se sentiam aptos a lidar com adolescentes ao terminar a sua formação pré-graduada (médicos: 93,9\%; enfermeiros: 87,7\%) (Quadro I). Em relação à formação recebida durante e após o internato de formação específica, a maioria dos médicos especialistas considerou ter recebido formação insuficiente durante o internato $(77,5 \%)$, não se sentir apto para lidar com adolescentes no final do mesmo $(68,4 \%)$ e ter recebido formação insuficiente após a conclusão do internato $(73,0 \%)$. Tanto os médi$\cos (100 \%)$ como os enfermeiros $(87,7 \%)$ concordaram ter necessidade de receber mais formação nesta área.

A maioria dos participantes referiu que preferia trabalhar com grupos etários mais novos em detrimento dos adolescentes (Quadro I).

\section{Limitações e necessidades de treino adicional}

Os profissionais de saúde dos cuidados de saúde primários referiram limitações nas diversas áreas de abordagem ao adolescente, sobretudo no que respeita à avaliação da família, dos problemas escolares e de comportamento e das queixas psicossomáticas e depressão (Quadro II). Os participantes referiram necessidade de receber treino adicional na maioria das áreas $(P<0,01)$. Mesmo referindo dificuldades em algumas áreas, alguns profissionais de saúde não sentiam ne- 
QUADRO I. Caracterização demográfica da amostra e opiniões acerca da formação pré e pós-graduada recebida em saúde do adolescente, por grupo profissional

\begin{tabular}{l|r|r} 
& Médicos $(n=33)$ & Enfermeiros $(n=57)$ \\
\hline Feminino $n$ (\%) & $27(81,8)$ & $56(98,2)$ \\
\hline Idade (anos) & $41,5 \pm 12,6$ & $42,8 \pm 7,8$ \\
\hline Grupo etário preferido - Adolescente $n$ (\%) & $13(39,4)$ & $17(29,8)$ \\
\hline Formação pré-graduada (1 a 7) & $2,4 \pm 1,2$ & $3,5 \pm 1,1$ \\
Durante a faculdade recebi formação suficiente em saúde do adolescente & $2,7 \pm 1,1$ & $3,3 \pm 1,0$ \\
Quando terminei a faculdade sentia-me apto a lidar com adolescentes & $5,8 \pm 0,8$ & $5,6 \pm 1,1$ \\
Preciso receber mais formação em saúde do adolescente & Médicos especialistas & $(n=25)$ \\
\hline & & - \\
\hline Formação pós-graduada (1 a 7) & $3,6 \pm 1,4$ & - \\
Durante o internato recebi formação suficiente em saúde do adolescente & $4,1 \pm 1,1$ \\
Quando terminei o internato sentia-me preparado para lidar com adolescentes & $3,6 \pm 1,1$ & - \\
Como especialista tenho recebido formação suficiente em saúde do adolescente &
\end{tabular}

\begin{tabular}{|c|c|c|}
\hline & $\begin{array}{c}\text { Sentem } \\
\text { Limitações (\%) }\end{array}$ & $\begin{array}{c}\text { Necessitam } \\
\text { Treino adicional (\%) }\end{array}$ \\
\hline Entrevista ao adolescente & 47,5 & 69,5 \\
\hline Problemas do desenvolvimento e crescimento & 26,3 & 38,1 \\
\hline Alimentação, obesidade e perturbações do comportamento alimentar & 16,9 & 33,9 \\
\hline Sexualidade, gravidez e comportamentos sexuais de risco & 28,0 & 49,2 \\
\hline Consumo de substâncias psicoativas & 45,8 & 63,6 \\
\hline Família: dinâmicas, adulto de referência e problemas familiares & 52,5 & 72,9 \\
\hline Problemas escolares e de comportamento & 54,2 & 62,3 \\
\hline Queixas psicossomáticas, depressão e risco de suicídio & 80,5 & 82,2 \\
\hline Comportamentos de risco e acidentes & 39,8 & 44,1 \\
\hline Especialista & $5(6 \%)$ & $10(13 \%)$ \\
\hline
\end{tabular}

cessidade de treino adicional nessas áreas (Quadro II). Da mesma forma, quando não havia referência a dificuldades, houve alguns profissionais de saúde que gostariam de receber formação adicional (Figura 1).

\section{DISCUSSÃO}

Os resultados deste estudo mostram que a maioria dos médicos e enfermeiros de família que frequentaram uma ação formativa considera não ter recebido formação adequada em saúde do adolescente. Este estudo identificou também as áreas da saúde do adolescente em que os profissionais consideram ter maiores dificuldades e necessidades formativas.

No nosso país, a maioria dos adolescentes é acom- 


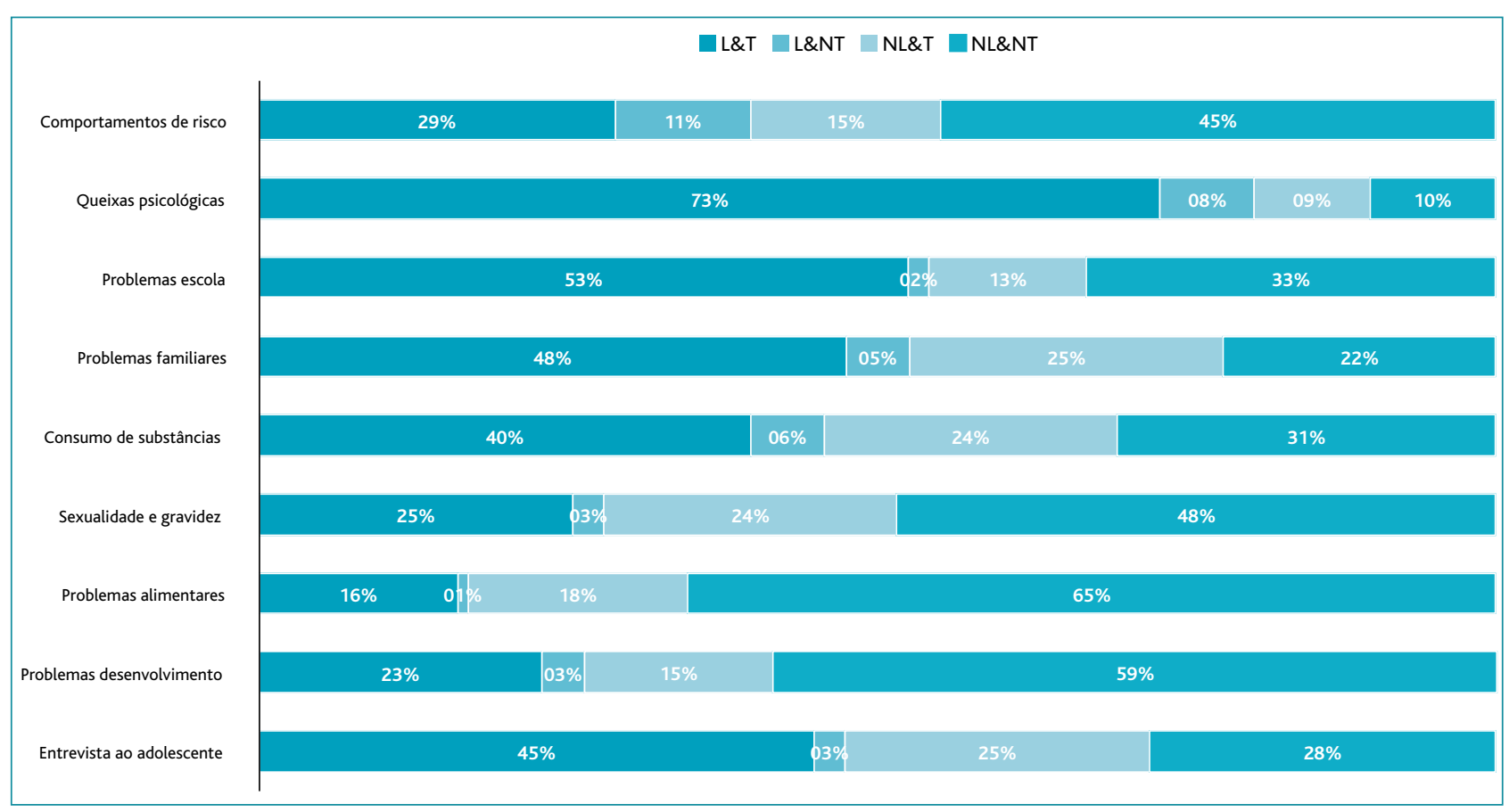

Figura 1. Cruzamento entre a necessidade de treino adicional e as limitações sentidas, por área de conhecimento.

Legenda: L\&T = Tenho limitação e necessito de treino adicional; L\&NT = Tenho limitação e não necessito de treino adicional; NL\&T = Não tenho limitação, mas necessito de treino adicional; NL\&NT = Não tenho limitação nem necessito de treino adicional.

panhada ao nível dos cuidados de saúde primários, pelo que seria expectável que estes profissionais recebessem formação adequada nesta área específica. No entanto, à semelhança de outros estudos, ${ }^{2,22,29}$ a maioria dos participantes considerou que a sua formação pré-graduada foi inadequada, não os deixando preparados para lidar com os problemas específicos dos adolescentes. De salientar que, tendo em conta a idade média dos formandos, a formação pré-graduada de grande parte dos mesmos teve lugar há mais de uma década, não se podendo excluir um viés de memória.

No caso específico dos médicos de família, a noção de falta de formação assume contornos ainda mais expressivos, uma vez que também consideram insuficiente tanto a formação recebida durante o internato como a frequentada após a conclusão do mesmo. Esta lacuna poderá constituir uma barreira importante à prestação de cuidados a este grupo etário, dado que uma formação insuficiente resulta frequentemente em desconforto na abordagem a aspetos importantes da saúde do adolescente. ${ }^{29}$

Os resultados em saúde infantil e juvenil têm tido ga- nhos inequívocos nas últimas décadas em consequência dos programas de saúde implementados e de ações concertadas intersetoriais. ${ }^{30}$ Porém, embora as crianças e adolescentes sejam mais saudáveis que no passado, ainda se confrontam com problemas de saúde causados por acidentes, doenças crónicas, abuso de tabaco e de outras substâncias, excesso de peso e obesidade, bem como doenças e perturbações mentais. ${ }^{30}$ Torna-se, então, essencial a identificação de estratégias para minorar estes problemas, que passarão seguramente pela formação dos profissionais de saúde nesta área. ${ }^{31}$

As áreas em que a maioria dos médicos e enfermeiros indicou sentir maiores limitações foram ao nível da avaliação da família, dos problemas escolares e de comportamento e das queixas psicossomáticas e depressão. Ainda que estes resultados sejam concordantes com outros já publicados, ${ }^{22,29,32}$ eles reforçam a necessidade de se repensar a formação. Esta necessidade torna-se ainda mais premente ao constatar-se que a maioria destes profissionais, teoricamente habilitados a lidar com problemas familiares, considera não se sentir 
preparada para abordar esta área específica. Esta poderá ser uma interessante linha de investigação para estudos futuros.

Outro resultado interessante deste estudo está relacionado com a discordância entre a perceção de limitações em determinadas áreas da saúde do adolescente e a vontade em receber mais formação nestas mesmas áreas: para qualquer uma das áreas formativas avaliadas foi sempre maior o número de inquiridos que indicaram necessitar de formação adicional do que o daqueles que indicaram sentir limitações; sugere a existência de vontade de alguns profissionais em aperfeiçoar as suas aptidões ainda que considerem não ter limitações numa determinada área. Uma outra possível explicação poderá relacionar-se com o facto de alguns profissionais, tendo limitações numa área, não o quererem reconhecer, estando, contudo, interessados em obter mais formação. Estes resultados contrastam com estudos já publicados onde se verificou que menos de um terço referiu interesse em prosseguir o desenvolvimento de competências necessárias para a abordagem às problemáticas de saúde dos adolescentes, apesar de muitos referirem treino insuficiente e que isso constituía uma barreira major ao atendimento ao adolescente. ${ }^{22,29}$ A problemática em que se verificou maior número de profissionais com limitações e sem interesse em receber formação foi a abordagem a comportamentos de risco, tendo-se cifrado nuns meros $11 \%$. Estes resultados mostram que os médicos e enfermeiros inquiridos têm interesse em receber mais formação na área da adolescência.

Para explicar as deficiências no atendimento ao adolescente é muitas vezes referido que os profissionais de saúde não gostam de lidar com adolescentes e evitam os problemas de saúde deste grupo etário. De facto, a maioria dos inquiridos neste estudo referiu que os adolescentes não eram o seu grupo etário preferido. Porém, este achado não se relacionou com perceção da formação que tinham recebido, nem com a sua vontade de obter mais treino nesta área. Como tal, e a par do retratado na literatura, o entrave ao atendimento ao adolescente não parece estar relacionado apenas com esta preferência. $^{22,29}$

O presente estudo apresenta algumas limitações. Trata-se de um estudo em que foi utilizada uma amostra de conveniência de profissionais de saúde que fre- quentaram uma ação de formação em saúde do adolescente e, como tal, os dados não podem ser inferidos para outras populações. Pode ainda argumentar-se que os profissionais de saúde que frequentaram estas ações de formação seriam os mais motivados e/ou os que teriam maior formação nesta área. Este argumento parece não se confirmar, já que a maioria dos profissionais inquiridos referiu que os adolescentes não eram o grupo etário pediátrico com o qual preferiam lidar.

Para minimizar o efeito do viés de seleção, os participantes foram escolhidos pelo coordenador de cada unidade e não através de acesso livre. Procurou abranger-se um grande número de profissionais de saúde e, apesar de não se incluírem profissionais de saúde das três Unidades Locais de Saúde da Região Centro, foram incluídos representantes dos seis ACeS da região. $\mathrm{O}$ questionário não foi testado previamente à sua aplicação, pelo que não se pode excluir um viés de informação. Por fim, trata-se de um estudo descritivo mas, considerando que se trata do primeiro do género em Portugal, poderá constituir um bom ponto de partida para estudos futuros. Torna-se claro com o presente estudo que os médicos e enfermeiros de família inquiridos consideram que a sua formação em saúde do adolescente, particularmente a pré-graduada, é insuficiente.

Fica ainda patente que, ao contrário do que tem sido relatado na literatura, os profissionais de saúde pretendem prosseguir a sua formação em saúde do adolescente e quais são as áreas em que se sentem com maiores limitações.

Esta informação poderá ser usada para melhor corresponder às necessidades de estudantes e de internos, criar um ambiente promotor de aprendizagem e desenhar currículos que sustentem o desenvolvimento de competências clínicas na provisão de cuidados aos adolescentes. Ao abordar estas questões, esperamos que o último objetivo de promover um cuidado de qualidade aos adolescentes e melhoria nos indicadores de saúde seja atingido.

\section{REFERÊNCIAS BIBLIOGRÁFICAS}

1. De Sanctis V, Clemente S, Gallotta M, Filati G, Fiscina B, Marsciani A, et al. The SGA-ER educational intervention in adolescent health care for Italian physicians: goals, content and instructional design. Georgian Med News. 2012;(210):13-8.

2. Diniz CS, Cunha CF, Ferreira RA. Medicina do adolescente: avaliação de uma experiência de ensino interdisciplinar [Adolescent medicine: eva- 
luation of an interdisciplinary teaching experimente]. Rev Med Minas Gerais. 2008;18(4 Suppl 1):S173-8. Portuguese

3. Direção-Geral da Saúde. Programa nacional de saúde infantil e juvenil: norma da Direção-Geral da Saúde n. ${ }^{\circ}$ 010/2013, de 31/05/2013.

4. Fonseca $\mathrm{H}$. Compreender os adolescentes: um desafio para pais e educadores. $4^{\mathrm{a}}$ ed. Lisboa: Editorial Presença; 2005.

5. Aratani Y, Schwarz SW, Skinner C. The economic impact of adolescent health promotion policies and programs. Adolesc Med State Art Rev. 2011;22(3):367-86.

6. Halfon N, DuPlessis H, Inkelas M. Transforming the U.S. child health system. Health Aff. 2007;26(2):315-30.

7. Center on the Developing Child. The foundations of lifelong health are built in early childhood [Internet]. Harvard University; 2010. Available from: https://46y5eh11fhgw3ve3ytpwxt9r-wpengine.netdna-ssl .com/wp-content/uploads/2010/05/Foundations-of-Lifelong-Health .pdf

8. Campbell KP. Investing in maternal and child health: an employer's toolkit [Internet]. Washington, DC: Center for Prevention and Health Services, National Business Group on Health; 2007. Available from: https://www.businessgrouphealth.org/pub/?id=f3004374-2354-d7145186-b5bc1885758a

9. Peixoto JC, Ferrão A, Duarte DG, Jonas P. Consulta dos $12-13$ anos (início da puberdade) [Internet]. Coimbra: ARS Centro; 2015. Available from: http://www.arscentro.min-saude.pt/Institucional/projectos/ crsmca/noc/Documents/saude \%20infantil/consulta\%201213\%20anosl.pdf

10. Klein D, Mehta K. Training in adolescent health: how much have secondyear residents had? Can Fam Physician. 2006;52(8):980-1.

11. American Academy of Family Physicians. Adolescent health: American Academy of Family Physicians. Am Fam Physician. 1999;60(2):660-2.

12. Neinstein LS, Shapiro JR. Pediatrician's self-evaluation of adolescent health care training, skills and interest. J Adolesc Health Care. 1986;7(1): 18-21.

13. PORDATA. População residente: total e por grupo etário [homepage]. Lisboa: PORDATA; 2017 Jun. Available from: http://www.pordata.pt/ Portugal/Populacao+residente+total+e+por+grupo+etario-10

14. Ozer EM, Adams SH, Gardner LR, Mailloux DE, Wibbelsman CJ, Irwin CE Jr. Provider self-efficacy and the screening of adolescents for risky health behaviors. J Adolesc Health. 2004;35(2):101-7.

15. Fisher M, Golden NH, Bergeson R, Bernstein A, Saunders D, Schneider $M$, et al. Update on adolescent health care in pediatric practice. J Adolesc Health. 1996;19(6):394-400.

16. Kershnar R, Hooper C, Gold M, Norwitz ER, Illuzzi JL. Adolescent medicine: attitudes, training, and experience of pediatric, family medicine, and obstetric-gynecology residents. Yale J Biol Med. 2009;82(4):129-41.

17. World Health Organization. Helping parents in developing countries improve adolescents' health. Geneva:WHO; 2007. ISBN 9789241595841

18. Garner AS, Shonkoff JP. Early childhood adversity, toxic stress, and the role of the pediatrician: translating developmental science into lifelong health. Pediatrics. 2012;129(1):e224-31.

19. Gupta RS, Shuman S, Taveras EM, Kulldorff M, Finkelstein JA. Opportu- nities for health promotion education in child care. Pediatrics. 2005;116(4):e499-505.

20. Hagan Jr JF, Shaw JS, Duncan PM, eds. Bright futures: guidelines for health supervision of infants, children, and adolescents. 3rd ed. Elk Grove Village, IL:American Academy of Pediatrics; 2008. ISBN 9781581102239

21. World Health Organization. Adolescent friendly health services: an agenda for change. Geneva:WHO; 2002.

22. Blum R. Physicians' assessment of deficiencies and desire for training in adolescent care. J Med Educ. 1987;62(5):401-7.

23. Consenso da Secção de Medicina do Adolescente. Requisitos para o atendimento ao adolescente. Acta Pediatr Port. 2005;36(4):223-4.

24. Hardoff D, Danziger Y, Reisler G, Stoffman N, Ziv A. Minding the gap: training in adolescent medicine when formal training programmes are not available. Arch Dis Child Educ Pract Ed. 2009;94(5):157-60.

25. Bravender T. Teaching adolescent medicine in the office setting. Curr Opin Pediatr. 2002;14(4):389-94.

26. Graves CE, Bridge MD, Nyhuis AW. Residents' perception of their skill levels in the clinical management of adolescent health problems. J Adolesc Health Care. 1987;8(5):413-8.

27. Fonseca H, Fernandes S, Coelho JP, Marcelino J, Gomes-Pedro J. Ensino pré-graduado de medicina da adolescência na Faculdade de Medicina de Lisboa: análise de 5 anos [Pre-graduate training in adolescent medicine at Faculdade de Medicina de Lisboa: a 5 year experience]. Acta Pediatr Port. 2004;35(3):267-72. Portuguese

28. Ribeiro C, Rosendo I. Saúde do adolescente em medicina geral e familiar [Adolescent health in family medicine]. Rev Port Clin Geral. 2011;27(2):184-6. Portuguese

29. Blum RW, Bearinger LH. Knowledge and attitudes of health professionals toward adolescent health care. J Adolesc Health Care. 1990;11(4): 289-94.

30. Machado MC, Alves MI, Couceiro ML. Saúde infantil e juvenil em Portugal: indicadores do Plano Nacional de Saúde [Child and adolescent health: Portuguese Health Plan indicators (2000-2010)]. Acta Pediatr Port. 2011:42(5):195-204. Portuguese

31. Patton GC, Sawyer SM, Santelli JS, Ross DA, Afifi R, Allen NB, et al. Our future: a Lancet commission on adolescent health and wellbeing. Lancet. 2016;387(10036):2423-78.

32. Klitsner IN, Borok GM, Neinstein L, MacKenzie R. Adolescent health care in a large multispecialty prepaid group practice: who provides it and how well are they going? West J Med. 1992;156(6):628-32.

\section{CONFLITO DE INTERESSES}

Os autores declaram a inexistência de quaisquer conflitos de interesse.

\section{ENDEREÇO PARA CORRESPONDÊNCIA}

Maria Inês Santos

E-mail: mines.santos82@gmail.com

http://orcid.org/0000-0002-0079-6366

Recebido em 24-03-2017

Aceite para publicação em 02-03-2018 
ABSTRACT

\section{ADEQUACY OF TRAINING IN ADOLESCENT HEALTH: OPINIONS OF FAMILY PHYSICIANS AND NURSES WHO ATTENDED A TRAINING COURSE}

Objectives: To assess the perceptions of primary care professionals on the training received in adolescent health, and to identify the perceived limitations and additional training needs.

Study design: Observational, cross-sectional and descriptive study.

Setting: Centre region.

Population: Family physicians and nurses of the Centre region.

Methods: The family physicians and nurses enrolled in an adolescent health training session were invited to answer a survey in which they were asked to evaluate their pre- and post-graduate training in adolescent health, the need to receive further training, and the limitations.

Results: Most participants considered that their pre-graduate training was insufficient (physicians: 97\%; nurses: 77.2\%) and did not feel able to deal with adolescents after graduation (physicians: $93.9 \%$; nurses: $87.7 \%$ ). The majority of family physicians also considered that the training received during residency was insufficient (77.5\%); they didn't feel able to deal with adolescents upon finishing residency (68.4\%), and considered that the training received after residency was also insufficient $(73.0 \%)$. Participants considered that they need additional training in adolescent health, pointing out areas with greater training needs: psychosomatic complaints (82.2\%), family problems (72.9\%), clinical interview (69.5\%), substance use (62.6\%), and school and behavioral problems $(62.3 \%)$.

Conclusions: The family physicians and nurses interviewed reported significant training gaps in adolescent health, both in their pre- and post-graduate training, as well as the need to obtain further training in this area.

Keywords: Education; Adolescent health; Primary health care 


\section{Questionário}

Sexo:

Masculino

Feminino

Idade:

\section{Formação:}

Especialista em medicina geral e familiar

Enfermeiro

Interno de medicina geral e familiar

De seguida encontra seis frases relacionadas com o treino em Saúde do Adolescente. Por favor, assinale com um círculo, numa escala de 1 a 7 (desde «Discordo totalmente» a «Concordo totalmente»), o seu nível de concordância em relação a cada uma:

\begin{tabular}{|c|c|c|c|c|c|c|c|}
\hline & $\begin{array}{c}\text { Discordo } \\
\text { totalmente }\end{array}$ & $\begin{array}{l}\text { Discordo } \\
\text { bastante }\end{array}$ & Discordo & $\begin{array}{l}\text { Não concordo } \\
\text { nem discordo }\end{array}$ & Concordo & $\begin{array}{l}\text { Concordo } \\
\text { bastante }\end{array}$ & $\begin{array}{c}\text { Concordo } \\
\text { totalmente }\end{array}$ \\
\hline $\begin{array}{l}\text { 1. Durante a faculdade recebi } \\
\text { formação suficiente em } \\
\text { medicina do adolescente }\end{array}$ & 1 & 2 & 3 & 4 & 5 & 6 & 7 \\
\hline $\begin{array}{l}\text { 2. Quando terminei a } \\
\text { faculdade sentia-me apto a } \\
\text { lidar com adolescentes }\end{array}$ & 1 & 2 & 3 & 4 & 5 & 6 & 7 \\
\hline $\begin{array}{l}\text { 3. Durante o internato } \\
\text { complementar recebi } \\
\text { formação suficiente em } \\
\text { medicina do adolescente }\end{array}$ & 1 & 2 & 3 & 4 & 5 & 6 & 7 \\
\hline $\begin{array}{l}\text { 4. Quando terminei a } \\
\text { especialidade sentia-me apto } \\
\text { a lidar com adolescentes }\end{array}$ & 1 & 2 & 3 & 4 & 5 & 6 & 7 \\
\hline $\begin{array}{l}\text { 5. Enquanto especialista } \\
\text { tenho recebido formação } \\
\text { suficiente em medicina do } \\
\text { adolescente }\end{array}$ & 1 & 2 & 3 & 4 & 5 & 6 & 7 \\
\hline $\begin{array}{l}\text { 6. Preciso de receber mais } \\
\text { formação em medicina do } \\
\text { adolescente }\end{array}$ & 1 & 2 & 3 & 4 & 5 & 6 & 7 \\
\hline
\end{tabular}

Em relação às seguintes dimensões, em quais considera ter limitações? (assinale com uma cruz)

1. Entrevista ao adolescente $\square$

2. Problemas do desenvolvimento e crescimento (físico, social e psicológico) $\square$

3. Alimentação, obesidade e perturbações do comportamento alimentar
4. Sexualidade, gravidez e comportamentos sexuais de risco $\square$

5. Consumo de substâncias psicoativas (tabaco, álcool e outras substâncias)

6. Família: dinâmicas, adulto de referência e problemas familiares $\square$

7. Problemas escolares e de comportamento $\square$

8. Queixas psicossomáticas, depressão e risco de suicídio

9. Comportamentos de risco e acidentes
Ordene de 1 a 3 os seguintes grupos pediátricos, de acordo com a preferência pessoal que tem no seu atendimento (sendo «1» para os que mais gosta de atender e « 3 » para os que menos gosta de atender):

Recém-nascido/Lactente

Criança

Adolescente

Em relação às seguintes dimensões, sobre quais gostaria de receber formação? (assinale com uma cruz)

1. Entrevista ao adolescente
2. Problemas do desenvolvimento e crescimento (físico, social e psicológico)

3. Alimentação, obesidade e perturbações do comportamento alimentar

4. Sexualidade, gravidez e comportamentos sexuais de risco $\square$

5. Consumo de substâncias psicoativas (tabaco, álcool e outras substâncias)

6. Família: dinâmicas, adulto de referência e problemas familiares

7. Problemas escolares e de comportamento $\square$

8. Queixas psicossomáticas, depressão e risco de suicídio

9. Comportamentos de risco e acidentes 